\title{
Maternal and perinatal outcomes of dichorionic diamniotic twin pregnancies diagnosed with vanishing twin syndrome: a retrospective analysis from a single clinical center
}

\author{
Hakan Timur 1, 2, Rifat Taner Aksoy ${ }^{1}$, Aytekin Tokmak', Burcu Timur ${ }^{3}$, \\ Bugra Coskun ${ }^{1}$, Dilek Uygur ${ }^{1}$, Nuri Danisman ${ }^{4}$ \\ ${ }^{1}$ Department of Obstetrics and Gynecology, Zekai Tahir Burak Women's Health Education and Research Hospital, \\ University of Health Sciences, Ankara, Turkey \\ ${ }^{2}$ Department of Perinatology, Ordu University Training and Research Hospital, Ordu, Turkey \\ ${ }^{3}$ Department of Obstetrics and Gynecology, Etlik Zubeyde Hanim Teaching and Research Hospital, \\ University of Health Sciences, Ankara, Turkey \\ ${ }^{4}$ Department of Obstetrics and Gynecology, School of Medicine, Acibadem Mehmet Ali Aydinlar University, Istanbul, Turkey
}

\begin{abstract}
Objectives: Multiple pregnancies are known to be associated with adverse maternal and perinatal complications. However, data regarding the outcomes of spontaneously reduced twin pregnancies are limited. In the current study we aimed to evaluate the consequences of the vanishing twin syndrome (VTS) in dichorionic diamniotic twin pregnancies for both mother and baby in our perinatal center.

Material and methods: A total of 711 pregnancies were included into the study. 51 cases of vanishing twin syndrome constituted Group 1, 235 cases of normal twins constituted Group 2, and 425 singleton pregnancies formed Group 3. The pregnancies that had multifetal reduction and monochorionic twinning were excluded from both study group and twin control group. The collected data were as follows: age, gravidity, parity, gestational week at birth, delivery route, birth weight, obstetric complications, and maternal and perinatal outcomes.

Results: No significant difference was observed between the groups regarding mean maternal age $(p>0.05)$. Mean birth weight, gestational age at birth and preterm birth ratio were significantly lower in the Group 2 when compared with Group 1 and Group 3 (all $p<0.001$ ). Adverse perinatal outcomes including very low birth weight (VLBV) and low Apgar scores were more common in Group $1(p<0.05)$, but no significant difference was found between the groups in terms of neonatal intensive care unit admission and perinatal mortality ratios $(p>0.05)$. Obstetric complications such as preeclampsia, gestational diabetes and intrauterine growth restriction were significantly higher in Group 2 than in Group 1 and Group 3 (all $p<0.05)$. However, severe maternal morbidities were similar among three groups $(p=0.141)$.

Conclusions: VTS is seems to be associated with VLBV and low Apgar scores. However, the incidence of severe maternal and perinatal morbidity and mortality in pregnancies with VTS is similar to other pregnancies.

Key words: vanishing twins, perinatal outcome, maternal morbidity, risk factors, very low birth weight.
\end{abstract}

Ginekologia Polska 2018; 89, 1: 30-34

\section{INTRODUCTION}

Twin pregnancies have steadily increased since the last decades in many countries. This increase is explained by two major causes that the increased use of assisted reproduction techniques and older maternal age [1]. The rate of twin births is reported to increase with the rate of $76 \%$ from 1980 to 2009 in the United States, and it reached a record high in 2014 and was 33.9 twins per 1,000 births [2]. 
Multiple pregnancies are known to be associated with adverse maternal and fetal complications. Preterm birth, antenatal bleeding problems, gestational hypertension, and preeclampsia occurs more often in multiple pregnancies [3]. As early as 1945, Stoeckel for the first time suggested that the occurrence of multiple gestations was greater than the birth rate of multiple gestations. This was attributed to the event that one or more of the fetuses might be reabsorbed by the cause of early gestational death without leaving any trace of evidence [4]. Vanishing twin syndrome (VTS) is described to be a spontaneous reduction of one of the fetuses' in-utero which partially or completely disappears during gestation [5]. The data regarding the spontaneous reduction of twin pregnancies are limited. However, spontaneous reduction was reported to be a common occurrence in multiple pregnancies [6]. The pregnancies which were initially 3 or more gestations and later reduced to singletons or twins were born earlier unreduced pregnancies with the same initial number of fetuses [7].

Although the precise mechanisms and pathophysiology are needed to be clarified, the underlined mechanism is thought to be chromosomal abnormalities of the low quality and non-enhancing embryo. Another postulated cause of VTS may be early implantation crowding, resulting in an unfavorable implantation site with insufficient utero-placental exchange. As it usually results in resorption of the embryo and gestational sac in early gestation, when fetal death occurs beyond the first trimester, a fetus papyrus may develop [8]. If twin sacs are detected on ultrasound vanishing of one fetus is expected with the rate of $27 \%$ of the assisted reproduction pregnancies and with the rate of $40 \%$ of spontaneously conceived pregnancies [5]. Well, the most striking question is that what the prognosis of the ongoing pregnancy is after loss of one of the twins.

The prognosis after vanishing twin was reported to be good regardless of chorionicity [5]. Some studies suggested that vanishing twin was associated with disseminated intravascular coagulation, intrauterine growth restriction (IUGR), very low birth weight (VLBW), low Apgar scores, brain damage of the co-twin and perinatal mortality, especially in monochorionics [9-13].

In the current study we aimed to evaluate the consequence of the vanishing twin phenomenon in dichorionic diamniotic twin pregnancies both for mother and baby in our perinatal center.

\section{MATERIAL AND METHODS}

This retrospective comparative study was carried out on patients diagnosed with VTS between January 2008 and December 2013 in Zekai Tahir Burak Women's Health Education and Research Hospital, Ankara, a tertiary level maternity hospital which is located in the capital of Turkey, after approval of local institutional review board (Decision date and number: 01/30/2015-15). Due to the retrospective design of our study, informed consent was not obtained. During the study period, 51 patients with VTS who fulfilled the inclusion criteria and were followed up appropriately were identified from the hospital database and medical records. Approximately five normal twin pregnancies and ten singleton pregnancies were randomly selected for each VTS patient as control groups. Singleton pregnancies were recruited from both high and low risk pregnancies managed in hospital during the study period. The admission diagnosis, laboratory values, operative records and baby files of all patients were carefully examined. VTS was defined as disappearance of one of the sacs or embryos of a pair of twins during the first trimester. Patients with insufficient or incomplete data on medical records were excluded from the study. The pregnancies that had multifetal reduction and monochorionic twinning were also excluded from the study. Both spontaneous pregnancies and IVF pregnancies were included in the study. The collected data were as follows: age, gravidity, parity, gestational week at birth, birth weight, delivery type, Apgar scores (at $1^{\text {st }}$ and $5^{\text {th }}$ minute), neonatal intensive care unit (NICU) admission, obstetric complications and finally maternal and fetal morbidity and mortality. The data were compared between the twin pregnancy groups and the control group.

Gestational ages were calculated according to the last menstrual period or first trimester measurement of crown-rump length. Recurrent pregnancy loss (RPL) was defined as the consecutive loss of two or more clinically recognized pregnancies prior to the $20^{\text {th }}$ week of gestation. It was found that fetal biometry and Doppler examination by ultrasonography and continuous external fetal monitoring by cardiotocography were carried out for each patient. Preeclampsia was defined as elevated blood pressure after 20 weeks of gestation ( $\geq 140 \mathrm{~mm} \mathrm{Hg}$ systolic or $\geq 90 \mathrm{~mm} \mathrm{Hg}$ diastolic) plus proteinuria (> $300 \mathrm{mg} / 24$ hours). The IUGR diagnosis was made based on serial fetal biometrics, where measured values or estimated fetal weight were below the 10th percentile. Women were diagnosed with gestational diabetes mellitus (GDM) if $\geq 2$ plasma glucose values on the $100 \mathrm{~g}$ oral glucose tolerance test exceeded the cut-off values at fasting, $1 \mathrm{~h}, 2 \mathrm{~h}$, and $3 \mathrm{~h}$ respectively. Polyhydramnios was defined as an amniotic fluid index (AFI) greater than $25 \mathrm{~cm}$ in the late second or third trimester whereas oligohydramnios was considered as $\mathrm{AFI}<5 \mathrm{~cm}$. With 12-hour dosing interval, $12 \mathrm{mg}$ betamethasone was administered to all pregnant women at or below 34 weeks of gestation for fetal lung maturation. The need for neonatal intensive care unit (NICU) was approved by a neonatologist for some cases due to respiratory distress, low birth weight $(<2500 \mathrm{~g})$ and VLBW $(<1500 \mathrm{~g})$. Low Apgar score was defined as being below 7 in the $1^{\text {st }}$ and $5^{\text {th }}$ minute. Severe 
maternal morbidity (e.g., postpartum bleeding, peripartum hysterectomy, need for blood transfusion, eclampsia, HELLP syndrome, acute renal failure, disseminated intravascular coagulation, adult respiratory distress syndrome, etc.) was defined as previously reported [14].

\section{Statistics}

Statistical Package for the Social Sciences version 15.0 (SPSS Inc., Chicago, IL, USA) was used for statistical analysis. The normality of the data was tested with Shapiro Wilk test. Descriptive statistics were used to demonstrate the mean \pm standard deviation or median (min-max) for constant variables, whereas nominal variables were expressed as case number and percentages. While fetal outcomes are calculated per infant in normal twin pregnancies, other outcomes are calculated per pregnancy. The continuous data was compared with the one way ANOVA and Kruskal-Wallis tests where suitable. Categorical data were compared with Chi-square test for trends and when expected value problem was present Fishers' exact test was used. A p value $<0.05$ was considered as statistically significant.

\section{RESULTS}

A total of 711 pregnancies were included into the study. 51 cases of vanishing twin group constituted Group 1, 235 cases of normal twins constituted Group 2, and 425 singleton pregnancies formed Group 3. Mean age in study and control groups were $28.5 \pm 5.3$ years, $28.5 \pm 6.0$ years, and $28.2 \pm 5.9$ years, respectively ( $p=0.853$ ). There was no significant difference between the groups in terms of median gravidity ( $p=0.096$ ) but the median parity was significantly lower in the normal twins than the other groups $(p=0.023)$. Three patients in Group 1, and 36 patients in Group 2 and ten patients in Group 3 conceived through in-vitro fertilization
(IVF), and a significant difference between the groups was observed $(p<0.001)$. Similarly, there was a significant difference between the three groups regarding the history of RPL ( $p=0.020)$. The comparison of maternal characteristics between the groups is depicted in Table 1. Birth weight was significantly higher in singleton pregnancy group than in normal twins and VTS groups $(p<0.001)$. Accordingly, gestational age at birth, and preterm birth ratio were significantly lower in Group 2 when compared with Group 1 and Group 3 $(p<0.001, p=0.001$, and $<0.001$, respectively). Second trimester bleeding was more common in VTS group $(p=0.016)$.

However, adverse perinatal outcomes such as very low birth weight and low Apgar scores were more frequent in the VTS group $(p<0.05)$. The comparison between the groups for maternal and perinatal characteristics is shown in the Table 2. There was a significant difference between the groups in terms of obstetric complications including preterm birth, preeclampsia, GDM, IUGR, premature rupture of membranes (PROM), and polyhydramnios (all $p<0.05$ ). However, no significant difference was observed between the groups in terms of fetal malformations $(p<0.05)$. NICU admission and perinatal mortality ratios were not statistically significantly different between the three groups (both $p>0.05$ ). In a similar manner, severe maternal morbidity was more frequent in the normal twins group, but the difference was not statistically significant among the groups $(p=0.141)$. No maternal mortality case occurred in any of the groups.

\section{DISCUSSION}

VTS describes a spontaneous reduction of a fetus while still in utero, which dissipates either partially or completely during gestation [5]. Early spontaneous reduction from twin to singleton pregnancy is common, and may be associated

\begin{tabular}{|l|c|c|c|c|}
\hline Table 1. Comparison of the demographics and obstetrical characteristics of the groups & \\
\hline & Vanishing twins (n:51) & Normal twins (n:235) & Singleton pregnancies (n:425) & $\mathbf{p}$ \\
\hline Age & $28.5 \pm 5.3$ & $28.5 \pm 6.0$ & $28.2 \pm 5.9$ & 0.853 \\
\hline Gravidity (median) & $2(1-5)$ & $2(1-7)$ & $2(1-6)$ & 0.096 \\
\hline Parity (median) & $1(0-3)$ & $0(0-5)$ & $1(0-5)$ & 0.023 \\
\hline Gestational age at birth & $36.3 \pm 2.3$ & $34.4 \pm 3.4$ & $38.2 \pm 2.3$ & 0.001 \\
\hline RPL & $2(3.1)$ & $12(5.1)$ & $6(1.4)$ & 0.020 \\
\hline IVF & $3(5.9)$ & $36(15.4)$ & $10(2.4)$ & $<0.001$ \\
\hline Birth weight & $2804 \pm 878$ & $2201 \pm 470$ & $3105 \pm 698$ & $<0.001$ \\
\hline Cesarean section & $21(41.2)$ & $227(96.6)$ & $195(45.9)$ & $<0.001$ \\
\hline Gender & & & & $232(54.6)$ \\
\hline \\
Male
\end{tabular}

$\mathrm{RPL}$ - recurrent pregnancy loss, IVF — in-vitro fertilization. Data are presented as mean \pm standard deviation, median (minimum-maximum) and number (percentage). A p value $<0.05$ is considered statistically significant 
Table 2. Comparison of the maternal and perinatal outcomes between the groups

\begin{tabular}{|c|c|c|c|c|}
\hline & Vanishing twins (n:51) & Normal twins ( $\mathrm{n}: 235)$ & Singleton pregnancies (n:425) & $\mathbf{p}$ \\
\hline Preterm birth (< 37 weeks) & $18(35.3)$ & $200(85.1)$ & $51(12)$ & $<0.001$ \\
\hline Late preterm birth (> 34 weeks) & $8(15.7)$ & $124(52.8)$ & $18(4.2)$ & $<0.001$ \\
\hline PROM (<37 weeks) & $8(15.7)$ & $40(17)$ & $9(2.1)$ & $<0.001$ \\
\hline $\begin{array}{l}\text { Intrauterine growth restriction } \\
\left(<10^{\text {th }} \text { percentile }\right)\end{array}$ & $5(9.8)$ & $33(14.1)$ & $24(5.6)$ & 0.001 \\
\hline Preeclampsia & $2(3.9)$ & $23(9.8)$ & $13(3.1)$ & 0.001 \\
\hline Chronic hypertension & 0 & $6(2.6)$ & $6(1.4)$ & 0.344 \\
\hline Gestational diabetes & $3(5.9)$ & $20(8.5)$ & $9(2.1)$ & $<0.001$ \\
\hline Polyhydramnios (AFI > $25 \mathrm{~cm}$ ) & 0 & $1(0.4)$ & $26(6.1)$ & $<0.001$ \\
\hline Oligohydramnios (AFI < $5 \mathrm{~cm}$ ) & $6(11.8)$ & $14(6)$ & $34(8)$ & 0.322 \\
\hline Second trimester bleeding & $6(11.8)$ & $5(2.1)$ & $20(4.7)$ & 0.016 \\
\hline Fetal malformation & 0 & $8(1.7)$ & $4(0.9)$ & 0.648 \\
\hline Malpresentation & $3(5.9)$ & $164(34.9)$ & $26(6.1)$ & 0.001 \\
\hline Low Apgar $1^{\text {th }} \min (<7)$ & $10(19.6)$ & $66(14)$ & $42(9.9)$ & 0.049 \\
\hline Low Apgar $5^{\text {th }} \min (<7)$ & $7(13.7)$ & $9(1.9)$ & $38(8.9)$ & $<0.001$ \\
\hline Macrosomia (> $4000 \mathrm{~g}$ ) & 0 & 0 & $9(2.1)$ & 0.009 \\
\hline Low birth weight $(<2500 \mathrm{~g})$ & $5(9.8)$ & $169(36)$ & $39(9.2)$ & $<0.001$ \\
\hline Very low birth weight $(<1500 \mathrm{~g})$ & $6(11.8)$ & $31(6.6)$ & $17(4.0)$ & 0.039 \\
\hline NICU admission & $6(11.8)$ & $38(8.1)$ & $30(7.1)$ & 0.475 \\
\hline Perinatal mortality & $1(2)$ & $8(1.7)$ & $3(0.7)$ & 0.366 \\
\hline Severe maternal morbidity & $1(2)$ & $21(8.9)$ & $26(6.1)$ & 0.141 \\
\hline
\end{tabular}

$\mathrm{PROM}$ - premature ruptures of membranes, AFI — amniotic fluid index, NICU — neonatal intensive care unit. Data are presented as mean \pm standard deviation, median (minimum-maximum), and number (percentage). A p value $<0.05$ is considered statistically significant

with an increased risk of late pregnancy complications [13]. In one study of 549 twin pregnancies, spontaneous reduction of one gestational sac or embryo occurred in about $36 \%$ of twin pregnancies until 12 weeks of gestation [7]. According to that study, VTS occurred in $27 \%$ of pregnancies diagnosed as twins prior to 7 weeks of gestation and both sacs were lost in $9 \%$ of them. Interestingly, studies have consistently shown that, in pregnancies conceived by IVF, the rate of early loss of the entire pregnancy is significantly lower for twin than singleton gestations [15].

Additionally, it is quite possible that the prevalence of spontaneous reduction is essentially much higher, since VTS may often be undetected throughout the course of pregnancy and labor. Although there is no conclusive hypothesis on the etiology of VTS, intrinsic abnormalities and infections are the most common postulated etiologies, and it is thought that chromosomal aberrations within the embryo are the major reason for failed conceptions or early fetal death $[5,16]$. The most significant aberration leading to embryonic loss is the first meiotic division resulting in non-disjunction and aneuploidy. Autosomal trisomies, including trisomies 13, 15, 16, 18 and 21, account for the most common spontaneous pregnancy losses [17]. In addition, there is a significant absence of autosomal monosomies in spontaneous human abortions, suggesting that these aberrations must be lethal to embryonic viability very early post-fertilization, in fact before the first missed menstrual period. There is a continuous controversy on whether any detrimental perinatal outcome concerning infants delivered after VTS exists at all $[13,18]$. A recent meta-analysis evaluating the effect of VTS on birth weight and extremely preterm delivery of the remaining fetus in IVF patients showed that a slight adverse effect of VTS might be present. But this study only compared VTS with singleton pregnancies [19]. In our study, we found that VLBW were more common in VTS pregnancies, and presumably in relation to this situation, 1 th and $5^{\text {th }}$ minute Apgar scores were found significantly lower in the VTS when compared with normal twins and singleton pregnancies. However, NICU admission rates, which may lead to worse perinatal outcomes and consequently perinatal mortality ratios, were similar to that in other pregnancies.

Moreover, another study that examined only IVF pregnancies compared the outcomes of singletons, twins, and VTS and found that indeed VTS was associated with poorer outcome when compared with singletons, although a better outcome was noted when compared with twins [20]. Our study showed that even if the preterm birth and low birth weight were more common in the normal twins, VLBW and 
low Apgar scores at $1^{\text {th }}$ and $5^{\text {th }}$ minute were more frequent in the VTS group. Moreover, pregnancies conceived with IVF were more frequent in the normal twins than in the vanishing twins. Our results are consistent with studies showing that the risk of developing VTS is lower in IVF pregnancies than in spontaneous pregnancies. However, it is well known that IVF pregnancies are associated with poor perinatal and neonatal outcomes. This may be associated with the low gestational age at birth in the normal twin group.

There are very limited studies conducted on the potential maternal and perinatal outcomes ofVTS. There is no clear consensus within the literature regarding obstetrics and perinatal outcomes of those pregnancies. VTS was found to be associated with an increased risk for congenital anomalies [21], vaginal bleeding and PROM as compared with other types of gestation [22]. Our results showed that there is no increased risk of fetal malformation in patients with VTS. Preterm birth, low birth weight, IUGR, and PROM were more common in normal twins than in pregnancies with VTS. Furthermore, GDM and hypertensive disorders were more commonly encountered in this group. On the other hand, second trimester bleeding, VLBW, low Apgar scores were more frequently observed in VTS group, but NICU admission and perinatal mortality ratios were similar to the other pregnancies.

The major limitations of this paper are its retrospective nature, being a single center study and having relatively small sample size, particularly regarding VTS group. However, addition of a normal twin pregnancy group increases its validity and reliability. Monochorionic twins are known to be associated with poorer perinatal outcomes than dichorionic twins. Herein we only included dichorionic twins in the study. Additionally, we did not exclude the IVF pregnancies which are known to be associated with adverse pregnancy outcomes.

In conclusion, although the etiology remains obscure and different pathophysiological mechanisms have been proposed, the VTS is by no means a rare occurrence in pregnancies conceived following assisted reproduction treatment. In this work, it was demonstrated that pregnancies diagnosed with VTS have some worse perinatal outcomes such as VLBW and low Apgar scores. However, preterm birth rates and mean birth weights are better in patients with VTS as compared with normal twin deliveries. Similarly, VTS seems not to be associated with severe perinatal morbidity and mortality. Although adverse maternal outcomes seen in VTS pregnancies were less frequent than in normal twin and singleton pregnancies, there is no significant difference between these three types of pregnancy. These situations should be taken into account when following pregnancies complicated with VTS.

\section{Conflict of interest}

None declared.

\section{REFERENCES}

1. Chauhan SP, Scardo JA, Hayes E, et al. Twins: prevalence, problems, and preterm births. Am J Obstet Gynecol. 2010; 203(4): 305-315, doi: 10.1016/j.ajog.2010.04.031, indexed in Pubmed: 20728073.

2. Hamilton BE, Martin JA, Osterman MJ, et al. Births: Final Data for 2014. Natl Vital Stat Rep. 2015; 64(12): 1-64.

3. Doyle P. The outcome of multiple pregnancy. Hum Reprod. 1996; 11 Suppl 4: 110-7; discussion 118, indexed in Pubmed: 9147114.

4. Levi S. Ultrasonic assessment of the high rate of human multiple pregnancy in the first trimester. J Clin Ultrasound. 1976; 4(1): 3-5, indexed in Pubmed: 814138.

5. Landy HJ, Keith LG. The vanishing twin: a review. Hum Reprod Update. 1998; 4(2): 177-183, indexed in Pubmed: 9683354.

6. Steinkampf MP, Whitten SJ, Hammond KR. Effect of spontaneous pregnancy reduction on obstetric outcome. J Reprod Med. 2005; 50(8): 603-606, indexed in Pubmed: 16220766.

7. Dickey RP, Taylor SN, Lu PY, et al. Spontaneous reduction of multiple pregnancy: incidence and effect on outcome. Am J Obstet Gynecol. 2002; 186(1): 77-83, indexed in Pubmed: 11810089.

8. POSNER AC, KLEIN MA. Fetus papyraceus: recognition and significance. Obstet Gynecol. 1954; 3(1): 106-110, indexed in Pubmed: 13120059.

9. Moore CM, McAdams AJ, Sutherland J. Intrauterine disseminated intravascular coagulation: a syndrome of multiple pregnancy with a dead twin fetus. J Pediatr. 1969; 74(4): 523-528, indexed in Pubmed: 5767341.

10. Melnick M. Brain damage in survivor after in-utero death of monozygous co-twin. Lancet. 1977; 2(8051): 1287, indexed in Pubmed: 73982.

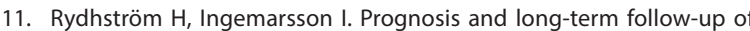
a twin after antenatal death of the co-twin. J Reprod Med. 1993; 38(2): 142-146, indexed in Pubmed: 8445607.

12. Okamura K, Murotsuki J, Tanigawara S, et al. Funipuncture for evaluation of hematologic and coagulation indices in the surviving twin following co-twin's death. Obstet Gynecol. 1994; 83(6): 975-978, indexed in Pubmed: 8190444.

13. Evron $\mathrm{E}$, Sheiner $\mathrm{E}$, Friger $\mathrm{M}$, et al. Vanishing twin syndrome: is it associated with adverse perinatal outcome? Fertil Steril. 2015; 103(5): 1209-1214, doi: 10.1016/j.fertnstert.2015.02.009, indexed in Pubmed: 25772775.

14. American Collage of Obstetrics and Gynecology. Obstetric Care Consensus No. 5: Severe Maternal Morbidity: Screening and Review. Obstet Gynecol. 2016; 128(3): e54-60.

15. La Sala GB, Villani MT, Nicoli A, et al. Effect of the mode of assisted reproductive technology conception on obstetric outcomes for survivors of the vanishing twin syndrome. Fertil Steril. 2006; 86(1): 247-249, doi: 10.1016/j.fertnstert.2005.11.073, indexed in Pubmed: 16716323.

16. Shebl $\mathrm{O}$, Ebner T, Sommergruber $\mathrm{M}$, et al. Birth weight is lower for survivors of the vanishing twin syndrome: a case-control study. Fertil Steril. 2008; 90(2): 310-314, doi: 10.1016/j.fertnstert.2007.06.048, indexed in Pubmed: 17931633.

17. Racowsky C. High rates of embryonic loss, yet high incidence of multiple births in human ART: is this paradoxical? Theriogenology. 2002; 57(1): 87-96, indexed in Pubmed: 11775983.

18. Tummers $P$, De Sutter P, Dhont M. Risk of spontaneous abortion in singleton and twin pregnancies after IVF/ICSI. Hum Reprod. 2003; 18(8): 1720-1723, indexed in Pubmed: 12871890.

19. Sun $L$, Jiang $L X, C$ hen $H Z$. Obstetric outcome of vanishing twins syndrome: a systematic review and meta-analysis. Arch Gynecol Obstet. 2017; 295(3): 559-567, doi: 10.1007/s00404-017-4289-9, indexed in Pubmed: 28097444.

20. Chasen ST, Luo G, Perni SC, et al. Are in vitro fertilization pregnancies with early spontaneous reduction high risk? Am J Obstet Gynecol. 2006; 195(3): 814-817, doi: 10.1016/j.ajog.2006.06.022, indexed in Pubmed: 16949417.

21. Pharoah POD, Glinianaia SV, Rankin J. Congenital anomalies in multiple births after early loss of a conceptus. Hum Reprod. 2009; 24(3): 726-731, doi: 10.1093/humrep/den436, indexed in Pubmed: 19059945.

22. Rodríguez-González M, Serra V, Garcia-Velasco JA, et al. The "vanishing embryo" phenomenon in an oocyte donation programme. Hum Reprod. 2002; 17(3): 798-802, indexed in Pubmed: 11870139. 